Research Paper

\title{
Establishment of metastatic liver carcinoma model by implanting AX7 cells into rabbit liver, and its histological findings
}

\author{
Sun Hyun Kim², Hyung Hwan Moon ${ }^{2}$, Myung Hee Yoon ${ }^{1 凶}$ \\ 1. Division of Hepatobiliarypancreas and Transplantation, Department of Surgery, Pusan National University Hospital, Busan, Republic of Korea \\ 2. Department of Surgery, Kosin University College of Medicine, Gospel Hospital, Busan, Republic of Korea \\ $\square$ Corresponding author: Myung Hee Yoon, Associate Professor, Biomedical Research Institute, Division of Hepatobiliarypancreas and Transplantation, \\ Department of Surgery, Pusan National University Hospital, 179 Gudeok-Ro, Seo-Gu, Busan, 49241 Republic of Korea. TEL: +82-51-240-7244; FAX: \\ +82-51-247-1365; E-Mail: ymh@pusan.ac.kr \\ (c) Ivyspring International Publisher. This is an open access article distributed under the terms of the Creative Commons Attribution (CC BY-NC) license \\ (https:// creativecommons.org/licenses/by-nc/4.0/). See http://ivyspring.com/terms for full terms and conditions.
}

Received: 2018.08.04; Accepted: 2018.12.11; Published: 2019.01.29

\begin{abstract}
Background: Progression of metastatic liver carcinoma from any original cancer is aggressive and the prognosis is very poor. Therefore the new model that is easily approachable to study the propagation and prognosis of metastatic liver carcinoma is necessary. The aim of this study is to confirm the tumor formation and metastatic activity of anaplastic thyroid cancer and to support the research basis for the next generation cancer treatment that is to be developed, by carrying out additional experiments like cytokine stimulation. We investigated sequential findings of immunohistochemistry of rabbit hepatic malignancy induced by $A \times 7$ cells.

Methods: 13 rabbits implanted with AX7 cells directly into liver parenchyme with laparotomy were investigated by histopathology examination, immunohistochemistry, which is useful for the evaluation of metastatic cancer angiogenesis. Growing tissue at the edge of the mass was collected and placed in the petri dish filled with saline. After removing necrotic and fibrous tissue, tumor tissue was cut into pieces, placed in saline, and extracted during the experiment.

Results: Tumor growth and malignancy was confirmed on the 10th day after AX7 cells were implanted into liver. Positive for VEGF staining was found in the cytoplasm or cell membrane. The scores for VEGF stained cells were moderately positive $(++)$ on day 10 , strongly positive $(+++)$ on day 44 . Ki-67-positive hepatocytes reached at $65 \%$ on day 10 , at $65.78 \%$ on day 14 , at $66.4 \%$ on day 30 , at $67.88 \%$ on day 44 .

Conclusion: AX7 cells implanted into liver can be used as a new rabbit metastatic liver carcinoma model and would become useful for human metastatic liver carcinoma studies. Future studies may facilitate the establishment of an effective systemic therapy for the metastatic liver cancer.
\end{abstract}

Key words: AX7 cells, Angiogenesis, metastatic liver carcinoma, rabbit

\section{Introduction}

Since its original description in 1933, VX2 has served as a surrogate for tumors involving the liver, esophagus, lung, kidney, uterus, head and neck (1-5). Because VX2 easily grows when implanted into the liver of rabbits, and develops into discrete lesions, this currently serves as the only hepatocellular carcinoma (HCC) model for animals.

Metastatic liver carcinoma is common from colon cancer, breast cancer, and HCC $(6,7)$. Human liver has a dual blood supply. A better understanding of its vascular supply and its hemodynamic changes may lead to early tumor detection. Angiogenesis is essential for the growth of primary and metastatic tumors, and it also alters vascular perfusion, and blood volume (8). The microvascular density (MVD) and vascular endothelial growth factor (VEGF) are used to clarify the physiological characteristics of angiogenesis and to detect easily the possibility of liver tumors. The pathological features of VX2 tumors are similar to those of human HCC (9). But, animal 
hepatic tumor cell line is not commonly available. So we tried to make rabbit hepatic tumor model using new cell line prepared and developed in animal laboratory of Kosin University and Pusan National University.

This study is a new AX7 hepatic tumor model for rabbits. Histology and immunohistochemistry were used to assess tumor growth and proliferation with VEGF and Ki-67. This paper is a step to confirm the tumor formation and metastatic activity of anaplastic thyroid cancer. The purpose of this paper is to identify the metastatic liver carcinoma and to support the research basis for the next generation cancer treatment that is to be developed, by carrying out additional experiments like cytokine stimulation.

\section{Materials and Methods}

Our study was carried out in strict accordance with the recommendations in the Guide for Care and Use of Laboratory Animal of the Kosin University. The protocol was approved by the Institution Animal Care and Use Committee at Kosin University (IRB Kosin 15-18) and all animal care and procedures were performed following institutional guidelines.

\section{Animals and $\mathbf{A X 7}$ cells injection}

13 New Zealand white rabbit, aged 8 months, weighed between $2.8 \mathrm{~kg}$ and $3.2 \mathrm{~kg}$ were used for this study. A suspension of AX7 cells (approximately $2 \times 10^{7}$ cells) was injected via a 26 gauge needle into the left lobe of the liver directly through small median subxyphoid incision under general anesthesia using zoletil $(0.3 \mathrm{mg} / \mathrm{kg})$ injection intramuscularly and isoflurane inhalation. The method of tumor implantation was followed as outlined by Liang et al (10). All of animals were euthanized at 7 days $(n=2)$, 10 days $(n=3), 14$ days $(n=3), 30$ days $(n=3)$, and 44 days $(n=2)$ after checking tumor growth.

\section{Hind Limb Tumor Harvest}

New Zealand white rabbits had AX7 samples inoculated into the hind limbs and served as donors for liver tumor implants and for propagation of the AX7 tumor strain. Donor rabbits were anesthetized with an intramuscular injection of zoletil $(0.3 \mathrm{mg} / \mathrm{kg})$. Bilateral hind limbs were shaved and disinfected with povidone-iodine $5 \%$. Then, tumors in the hind limbs were explanted and the rabbit was euthanized with $\mathrm{CO}_{2}$ air. One of the tumors was immediately processed, and the other was placed in normal saline for eventual liver tumor implantation.

In order to process the tumor for propagation, the AX7 tumor specimen was placed in a petri dish and the excess tissue removed. The tumor was rinsed with RPMI 1640 media (LifeTechnologies, Brand Island, NY) and the necrotic portions discarded.
Using a surgical blade, tumor cells were scrapped from the surrounding tissue. The cells were then filtered through a 40 micrometer mesh strainer (BD Biosciences, San Jose, CA) and centrifuged into a pellet. The supernatant was discarded and the cells were re-suspended in a 1:1 ratio with methylcellulose (StemCell Technologies, British Columbia, and Canada) and kept on ice. Cells were re-suspended in saline at $2 \times 10^{7}$ cells $/ 1 \mathrm{ml}$.

\section{Preparation of $\mathbf{A X 7}$ cell line}

AX tumor cells were provided by Professor Oak (Department of Internal Medicine, Division of Pulmonology, Kosin University, College of Medicine, Korea). AX7 tumor cells were originated from thyroid cancer. We named those cells AX7. The recovered tumor cells were inoculated into the muscle at rabbit' groin area. After 14 days, a solid mass was palpable at the inoculation site. The tumor was then dissected under anesthesia. Growing tissue at the edge of the mass was collected and placed in the petri dish filled with saline. After removing necrotic and fibrous tissue, tumor tissue was cut into $1.3 \mathrm{~mm} \times 1.3 \mathrm{~mm} \times 4$ $\mathrm{mm}$ size pieces, placed in saline, and extracted with 1-ml syringe connected with needle (diameter of 1.54 $\mathrm{mm}$ ) during the experiment to inject directly into liver parenchyme. I injected the cell suspensions in the middle anterior segment of liver with laparotomy to assure if the cell suspensions were in the same segment of liver.

\section{Histological examination}

The area in each of the 13 rabbits that received AX7 tumor implants was identified for microscopic examination. The rabbits were killed by $\mathrm{CO}$ air inhalation. The liver tissue was fixed in $10 \%$ formaldehyde and embedded in paraffin prior to histological and immunohistochemical study. Consecutive $4 \mu \mathrm{m}$ sections were cut and mounted on glass slides. Sections were stained for histological evaluation using standard haematoxylin and eosin staining.

\section{Evaluation of VEGF expression}

Tissue specimens from all hepatic tumors were fixed with $10 \%$ formalin, embedded with paraffin, and cut into $5 \mu \mathrm{m}$ thick slices. Immunohistochemistry assay was performed to determine the expression level of VEGF in tumor thrombus. Immunohistochemistry staining was conducted according to the kit instruction. The concentration of VEGF antibody was 1:300. VEGF positive staining was primarily found in the cytoplasm or cell membrane. For each slice, 5 high power fields (hpf) were randomly selected; semi-quantitative integral method was used to determine the results (11). The final score was 
calculated based on the staining intensity and the percentage of positive cells among the total number of tumor cells. Staining intensity was scored as follows: 0 for colorless, 1 for amber, 2 for brown, and 3 for tan. The number of positive cells was scored as follows: 0 if less than $10 \%$ of the total cells, 1 if $10 \%$ to $20 \%, 2$ if $21 \%$ to $50 \%$ and 3 if greater than $50 \%$. The scores for staining intensity and positive cells were then added together to determine the final result: negative if total score was 0 , weak positive $(+)$ if $1-3$, moderately positive $(++)$ if $4-5$, strongly positive $(+++)$ if the total score was greater than or equal to 6 .

\section{Hepatocyte Proliferation}

In assessing hepatocyte proliferation, liver tissues from the hepatic lobes were immune stained with anti-Ki-67 antibodies (monoclonal mouse anti-human Ki-67 antigen; MIB-5; Dako, Carpinteria, California), which detects all active parts of the cell cycle and has a strong positive correlation with proliferating antigen expression, bromodeoxyuridine incorporation, and thymidine incorporation (12). The immune stained sections were counterstained with hematoxylin. Ki-67-positive cells were counted in six medium-power fields (x200 magnification) per section. The proliferation index was defined as the percentage of Ki-67-positive hepatocytes per total hepatocyte count in the field of view. The criteria of nuclear labeling index for Ki67 in this study were as follows: four degrees of positive nuclei for Ki67 were identified: negative if $<24 \%$, weak positive $(+)$ if $25-\% 50 \%$, moderately positive (++) if $51 \%-74 \%$, strongly positive $(+++)$ if the total score was greater than or equal to $75 \%$.

\section{Statistical analysis}

All data were analyzed with SPSS (V17.0). The quantitative data were summarized with mean \pm standard deviation, and 95\% confidence interval. P $<0.05$ was defined as statistically significant.

\section{Results}

\section{Growth curve and microscopic morphology of $\mathrm{AX7}$ cells in culture}

(A)

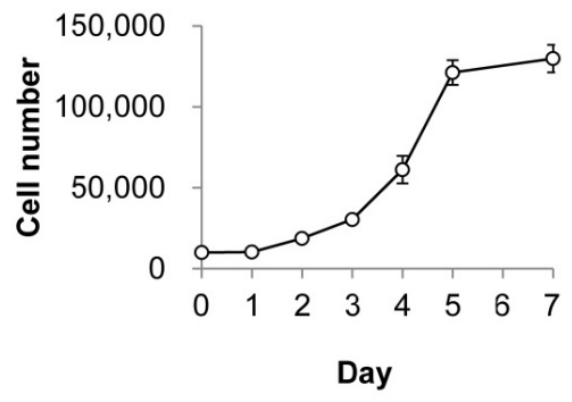

(B)

Day 2

Day 4
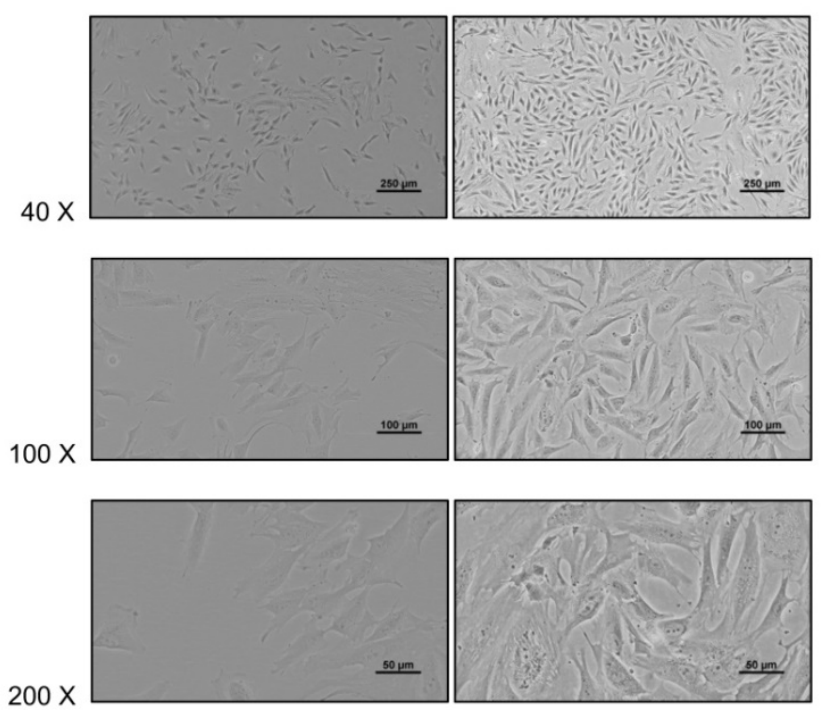

Figure 1. Growth curve (A) and microscopic morphology (B) of AX7 cells in culture. (A) Cells ( 10,000 cells/well) were plated in 12-well plates and viable cells were counted every day. Data are presented as mean \pm S.D. of duplicates. (B) Microscopic observation of AX7 cells on day 2 and 4 is shown. Scale bar: $250 \mu \mathrm{m}, 100 \mu \mathrm{m}$ and $50 \mu \mathrm{m}$ for $40 x$, $100 \times$ and $200 \times$ magnification, respectively. 


\section{Hepatic tumor growth by $\mathbf{A X 7}$ cell implantation}

A total of 13 New Zealand white rabbits underwent direct injection of AX7 cells into the liver under laparotomy (Fig 2). Hepatic tumor was grown in 11 rabbits $(85 \%, 11 / 13)$. Gross pathology (Fig 3) indicated that of the 13 rabbits, of them, 2 had metastases to other sites, primarily to the omentum, peritoneal cavity. Of the 13 rabbits the mean hepatic tumor size was $1.08 \mathrm{~cm}$ on day $10,1.47 \mathrm{~cm}$ on day 14 , $1.68 \mathrm{~cm}$ on day 30 and $2.35 \mathrm{~cm}$ on day 44 (Table 1 ).

\section{Histology and immunohistochemistry}

After the rabbits were sacrificed, gross pathology and histology was performed. Liver histology demonstrates a heterogeneous appearance to the tumor (Fig 4,5).

\section{VEGF expression and Hepatocyte Proliferation}

Positive for VEGF staining was found in the cytoplasm or cell membrane. The scores for VEGF stained cells were moderately positive $(++)$ on day 10 , strongly positive $(+++)$ on day 44 (Table 2) (Fig 6). Hepatocyte proliferation from the Lt hepatic lobes was evaluated by anti-Ki-67 staining. Ki-67-positive hepatocytes reached at $65 \%$ on day 10 , at $65.78 \%$ on day 14 , at $66.4 \%$ on day 30 , at $67.88 \%$ on day 44 (Table 2) (Fig 7).
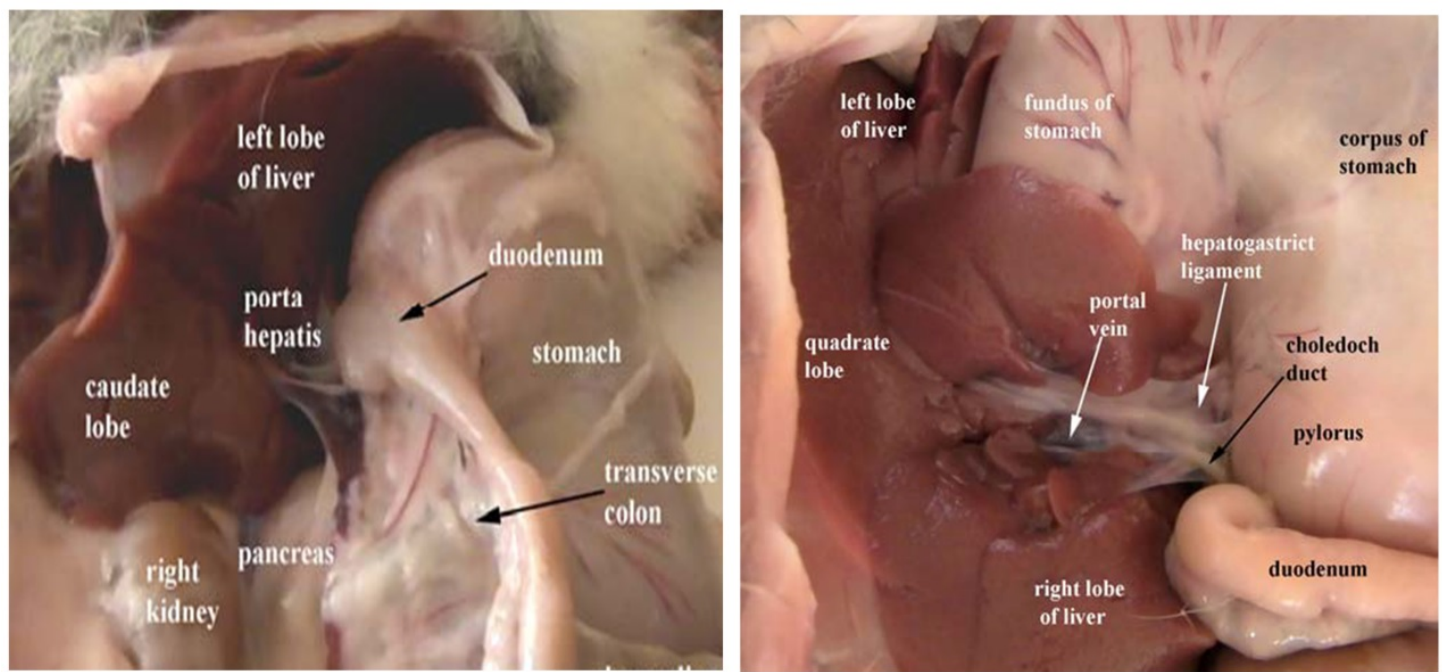

Figure 2. Anatomical topographic localization of the rabbit liver
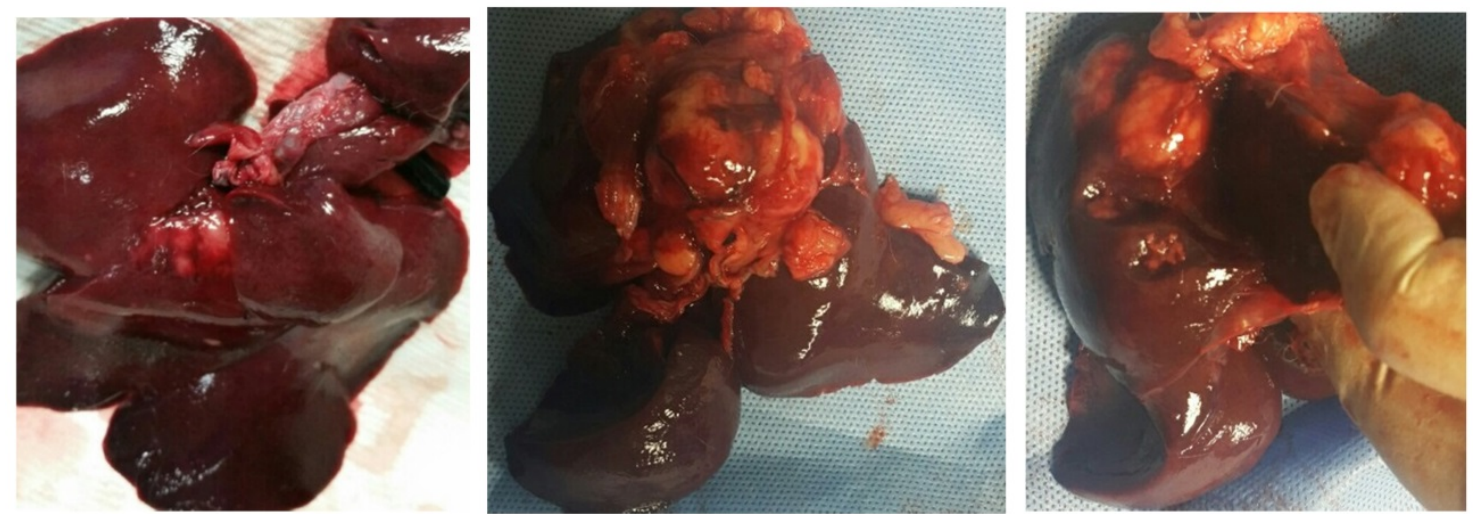

Figure 3. Gross findings. Explanted Liver lobes reveal tumors on 14th day $(A)$ and 30th day $(B, C)$ after $A X 7$ cells implantation.

Table 1. Mean tumor size.

\begin{tabular}{lllll}
\hline Post operative day $(\mathrm{POD})$ & POD 7 $(\mathrm{n}=2)$ & POD 10 $(\mathrm{n}=3)$ & POD 14 $(\mathrm{n}=3)$ & POD 30(n=3) \\
\hline Mean tumor size $(\mathrm{cm})$ & No tumor growth & 1.1 & 1.5 & POD 44(n=2) \\
\hline
\end{tabular}



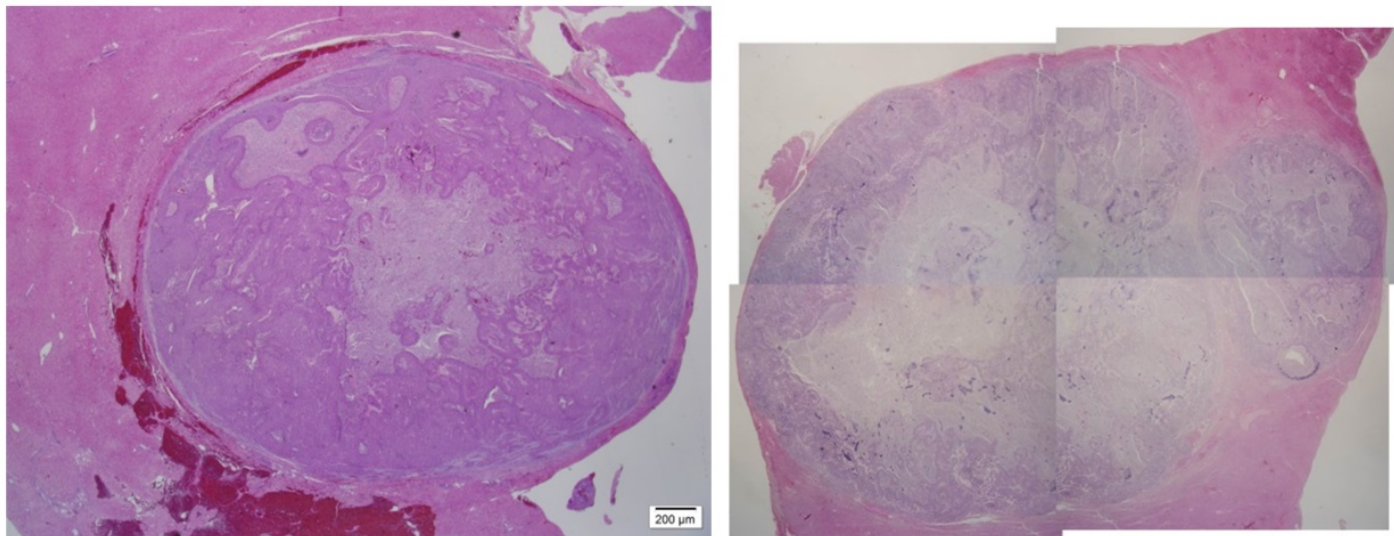

Figure 4. Histology. Hematoxylin \& Eosin staining was performed confirming tumors within the liver on 10th day (H\&E $\times 12.5)(A)$, and 44th day (H\&E $\times 6.5)(B)$.
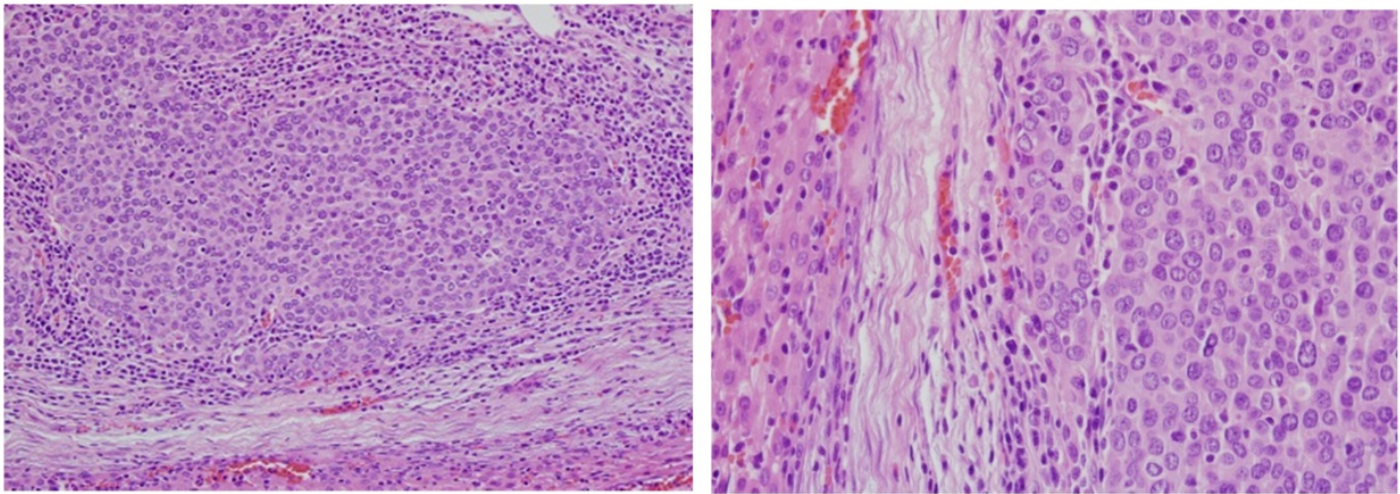

Figure 5. Histology. Hematoxylin \& Eosin staining was performed confirming tumors within the liver on 10th day (H\&E $\times 200)(A)$, and 10th day $(H \& E \times 400)(B)$.
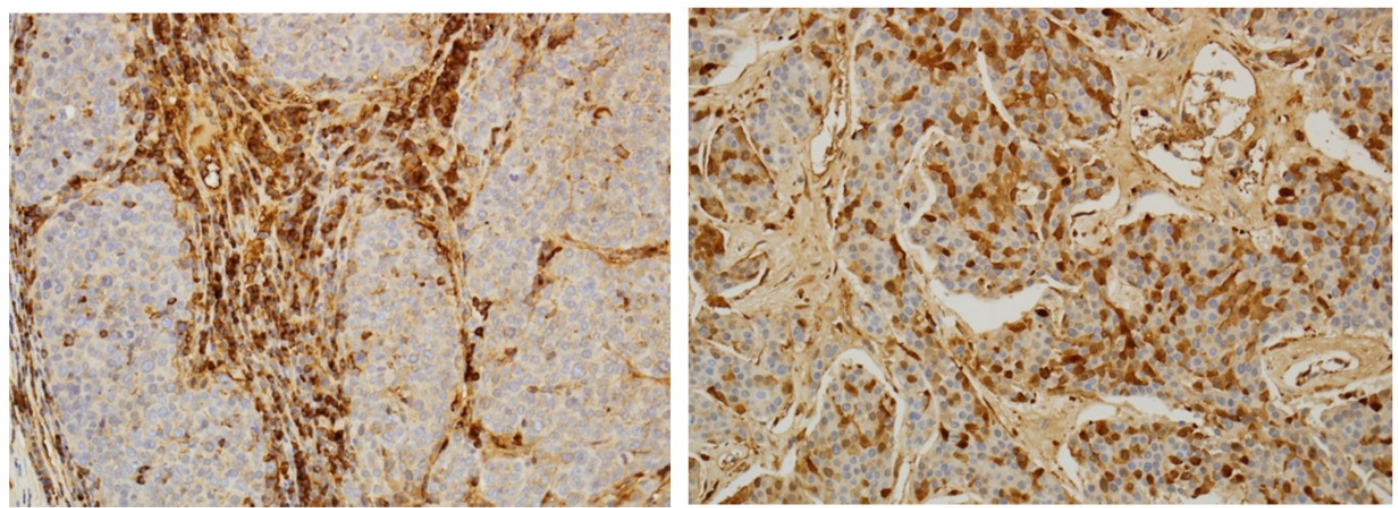

Figure 6. Immunohistochemical staining for VEGF shows the cytoplasm of tumor cells stained brown (dark) on 10th day $(\times 400)(A)$, and 44th day $(\times 400)(B)$.
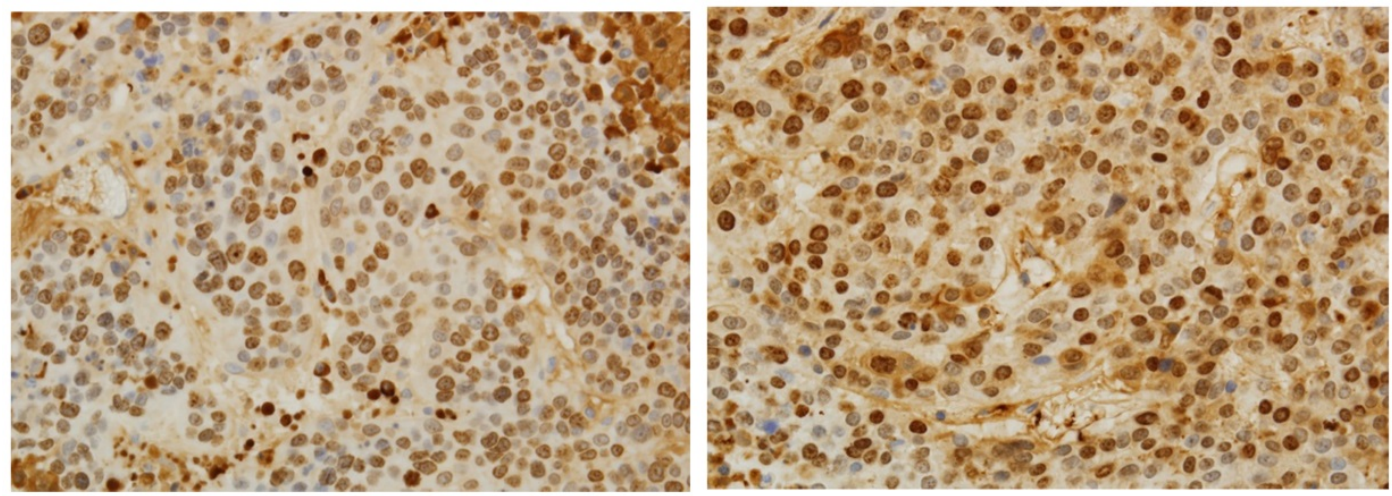

Figure 7. Detection of hepatocyte proliferation by anti-Ki-67 staining on $20^{\text {th }}$ day $(\times 400)(A)$, and $44^{\text {th }}$ day $(\times 400)(B)$. 
Table 2. Immunohistochemical staining score for VEGF and Ki-67 according to post operative day

\begin{tabular}{lllll}
\hline $\begin{array}{l}\text { Post Operative Day (POD)/ Rabbit } \\
\text { number }\end{array}$ & $\begin{array}{l}\text { Mean tumor size } \\
(\mathrm{cm})\end{array}$ & VEGF & Ki-67 (\%) \\
\hline POD 7 & 1 & no growth & none & none \\
& 2 & no growth & none & none \\
POD 10 & 3 & 0.95 & ++ & 65.00 \\
& 4 & 1.20 & ++ & 64.50 \\
& 5 & 1.10 & ++ & 65.50 \\
POD 14 & 6 & 1.45 & ++ & 65.00 \\
& 7 & 1.30 & ++ & 65.25 \\
& 8 & 1.65 & +++ & 67.10 \\
POD 30 & 9 & 1.70 & ++ & 65.40 \\
& 10 & 1.65 & +++ & 66.50 \\
& 11 & 1.70 & +++ & 67.30 \\
POD 44 & 12 & 2.40 & +++ & 68.25 \\
& 13 & 2.30 & +++ & 67.50 \\
\hline
\end{tabular}

\section{Discussion}

This paper is a step to confirm the tumor formation and metastatic activity by implanting the AX7 cell. The AX7 cell line was originated from anaplastic thyroid cancer, so it is a metastatic liver cancer model. The strong part of the AX7 cell is that it is injected right into the liver parenchyme under laparotomy and is approved of intrahepatic carcinoma formation. Then, we did find the histological features of the metastatic carcinogenic activity. But we did not intend to show any further characterization of the cells with respect to chemosensitivity or reaction towards cytokines.

The VX2 tumor is a squamous cancer of the skin induced by Shope virus (9). It shows a high rate of successful implantation, a stable history after implantation and the capacity for neovascularization, characteristics that make it suitable for liver imaging studies, especially studies of angiogenesis in liver tumors. As the tumor grows, the central portion becomes relatively hypovascular and eventually necrotic $(13,14)$. This may be seen as an image enhancement on conventional contrast enhanced CT.

Tumor sizes and growth rates were similar in this cohort as with prior studies reported in the literature. In addition, the tumor heterogeneity see in our histology is similar to histology sample seem from other implantation methods. Studies over the last decade have demonstrated that although the micro vessel density in VX2 tumors in heterogeneous, the vasculature of the tumor may differ substantially at different stages of growth. At the earlier stages of the tumor's growth, more blood vessels were shown in the peripheral rim of the tumor. This could explain the rapid tumor growth when tumor fragments are implanted (15).

The current methods of tumor implantation include surgical exposure of the liver, with surgical implantation of tumor fragments, open surgical implantation of VX2 cell suspension and percutaneous injection of minced tumor cells into the liver (16). Open surgical implantation of tumor fragments has proven to be the superior method, with studies reporting success rates ranging from $84--100 \%$, compared to $35--47 \%$ success rates when inoculating with cellular suspensions (16-18). Because of poor success rates with cell suspension inoculations, other groups developed ultrasound (US) guided methods of implantation with minced tumor cells, and showed 100\% technical success rates with decreased associated morbidities with their minimally invasive techniques (19-24).

Intraparenchymal implantation of $\mathrm{VX} 2$ is a widely accepted mechanism for creating a model of liver cancer in rabbits. Assessing tumor response with diffuse hepatic involvement can be very challenging. Therefore, injection of VX2 directly into the liver parenchyma has become the preferred method of tumor implantation. In previous work, tumor inoculation with cell suspension yielded only $35-47 \%$ success rates (16-19). This method was plagued with morbidity and mortality and leakage of tumor cells was reported to be as high as $50 \%$ and mortality rates of $22 \%$ were reported (16-19). Surgical implantation of tumor fragments resulted in higher rates of success in tumor growth at $84-100 \%$ (20-22). Ultrasound guided tumor implantation was successful in all 27 rabbits. One rabbit died 2 days following the implantation procedure. Two rabbits had no tumors seen on follow-up imaging. Therefore, tumor development was seen in 24/26 (92\%) rabbits (25). During the follow-up period, tract seeding was seen in $8 \%$ of rabbits and $38 \%$ had extra-hepatic metastatic disease (26-27). Therefore, percutaneous ultrasound guided tumor implantation safely provides reliable tumor growth for establishing hepatic VX2 tumors in a rabbit model with decreased rates of tract seeding, compared to previously reported methods.

The AX7 cell line was originated from thyroid cancer, so it is a metastatic liver cancer model. The established VX2-model is originated from "unspecified tissue origin", but it has been shown that the cells behave in many aspects like an HCC model, i.e. HEP-G2 (28). The result of the reference says that the two cellular models for HCC share several features related to sorafenib application, but differed in their responsiveness towards growth factors. Therefore, results obtained with the VX2 model cannot be extended to human HCC without appropriate caution.

This study has an obvious origin of cells. But, there were several limitations in our study. First, this study only includes a small number of rabbits $(n=13)$. Second, because this technique required fewer resources, it was used exclusively for tumor 
implantation; therefore, no open surgical group was available for comparison. As a matter of fact, we are planning our next experiment focused on this part, stimulation with cytokines that are present or sensitivity to chemotherapeutics for the treatment of metastatic liver carcinoma.

\section{Conclusion}

AX7 cell implantation into liver could be used as a new rabbit metastatic hepatic tumor model and would be useful to study human metastatic liver carcinoma. Future studies may facilitate the establishment of an effective systemic treatment for metastatic liver cancer.

\section{Acknowledgements}

There are no financial or other kinds of relationship that might lead to a conflict of interest. This work was supported by Pusan National University Research Grant, 2016.

\section{Competing Interests}

The authors have declared that no competing interest exists.

\section{References}

[1] Shope RE, Hurst EW. Infectious Papillomatosis of Rabbits: With a Note on the Histopathology. J Exp Med 1933;58:607-624.

[2] Geschwind JF, Artemov D, Abraham S et al. Chemoembolization of liver tumor in a rabbit model: assessment of tumor cell death with diffusion-weighted MR imaging and histologic analysis. JVIR 2000;11:1245-255.

[3] Larson AC, Rhee TK, Deng J, et al. Comparison between intravenous and intraarterial contrast injections for dynamic 3D MRI of liver tumors in the VX2 rabbit model. J Magn Reson Imaging 2006;24:242-247.

[4] Lee JM, Kim SW, Chung GH, et al. Open radio-frequency thermal ablation of renal VX2 tumors in a rabbit model using a cooled-tip electrode: feasibility, safety, and effectiveness. Eur Radiol 2003;13:1324-1332.

[5] Rhee TK, Ryu RK, Bangash AK, et al. Rabbit VX2 tumors as an animal model of uterine fibroids and for uterine artery embolization. JVIR 2007;18:411-418.

[6] Abou-Alfa GK, Schwartz L, Ricci S, et al. Phase II study of sorafenib in patients with advanced hepatocellular carcinoma. J Clin Oncol 2006;24:4293-4300.

[7] Llovet JM, Ricci S, Mazzaferro V, et al. Sorafenib in advanced hepatocellular carcinoma. N Engl J Med 2008;359:378-390.

[8] Purdie TG, Henderson E, Lee TY. Functinal CT imaging of angiogenesis in rabbit VX2 soft tissue tumor. Phys Med Biol 2001;46:3161-3175.

[9] Nasseri M, Wettstein FO. Differences exist between viral transcripts in cottontail rabbit papillomavirus-induced benign and malignant tumors as well as non-virus-producing and virus-producing tumors. J Virol 1984;51: 706-712.

[10] Liang B, Zheng C, Feng G, et al. Experimental evaluation of inhibitory effect of 10-hydroxycamptothecin on hypoxia-inducible factor-1expression and angiogenesis in liver tumors after transcatheter arterial embolization. J Vasc Interv Radiol 2010;21:1565-1572.

[11] Wallace S, Kan Z, Li C. Hepatic chemoembolization: clinical and experimental correlation. Acta Gastroenterol Belg 2000;63:169-173.

[12] Gerlach C, Sakkab DY, Scholzen T, et al. Ki-67 expression during rat liver regeneration after partial hepatectomy. Hepatology 1997;26:573-578.

[13] Stewart EE, Chen X, Hadway J, et al. Correlation between hepatic tumor blood flow and glucose utilization in a rabbit liver tumor model. Radiology 2006;239:740-750.

[14] Maruyama H, Matsutani S, Saisho H, et al. Sonographic shift of hypervascular liver tumor on blood pool harmonic images with definity: time-related changes of contrast-enhanced appearance in rabbit VX2 tumor under extralow acoustic power. Eur J Radiol 2005;56:60-65.

[15] Chen JH, Lin YC, Huang YS, Chen TJ, Lin WY, Han KW. Induction of VX2 carcinoma in rabbit liver: comparison of two inoculation methods. Lab Anim 2004;38(1):79-84

[16] Lin WY, Chen J, Lin Y, Han K. Implantation of VX2 carcinoma into the liver of rabbits: a comparison of three direct-injection methods. J Vet Med Sci 2002;64(7):649-652
[17] Virmani S, Harris KR, Szolc-Kowalska B, Paunesku T, Woloschak GE, Lee FT, et al. Comparison of two different methods for inoculating VX2 tumors in rabbit livers and hind limbs. Journal of vascular and interventional radiology 2008;19(6):931-936.

[18] Lee KH, Liapi E, Buijs M, Vossen JA, Prieto-Ventura V, Syed LH, et al. Percutaneous US-guided implantation of Vx-2 carcinoma into rabbit liver: a comparison with open surgical method. J Surg Res 2009;155(1):94-99.

[19] Parvinian A, Casadaban LC, Gaba RC. Development, growth, propagation, and angiographic utilization of the rabbit VX2 model of liver cancer: a pictorial primer and "how to" guide. Diagn Interv Radiol 2014;20(4):335-340.

[20] Wang Y, Xiong B, Liang B, et al. Hepatic parenchymal changes following transcatheter embolization and chemoembolization in a rabbit tumor model. PLoS ONE 2013;8:e70757.

[21] Shengli D, Qibin T, Miaoyun L, et al. The cooperative effect of p53 and Rb in local nanotherapy in a rabbit VX2 model of hepatocellular carcinoma. International J Nanomedicine 2013;8:3757-3768.

[22] Choi SH, Chung JW, Kim HC, et al. The Role of perfusion CT as a follow-up modality after transcatheter arterial chemoembolization: an experimental study in a rabbit model. Invent Radiol 2010;45:427-436.

[23] Wei C, Xiang X, Juliang Z, et al. Tumor angiogenesis after heated lipiodol Infusion via the hepatic artery in a rabbit model of VX2 liver. PLoS One 2013;8:e61583.

[24] Dong S, Tang Q, Long M, Guan J, Ye L, Li G. The cooperative effect of p53 and $\mathrm{Rb}$ in local nanotherapy in a rabbit VX2 model of hepatocellular carcinoma. International J Nanomedicine 2013;8:3757-3768.

[25] White SB, Chen J, Gordon AC, et al. Percutaneous ultrasound guided implantation of VX2 for creation of a rabbit hepatic tumor model. PLoS One 2015;10:e0123888

[26] Zhang YW, Ao J, Liu Y, et al. Pharmacokinetics of gelatin sponge microparticles in a rabbit VX2 liver tumor model of hepatic arterial chemoembolization. Tumour Biol 2014:35:10905-10910.

[27] Jiang HJ, Lu HB, Zhang ZR, et al. Experimental study on angiogenesis in a rabbit VX2 early liver tumour by perfusion computed tomography. J Int Med Res 2010;38:929-939.

[28] Nass N, Streit S, Wybranski C, et al. Validation of VX2 as a Hepatocellular Carcinoma Model: Comparison of the Molecular Reaction of VX2 and HepG2 Tumor Cells to Sorafenib In Vitro. Anticancer Res 2017;37(1):87-93. 